\title{
EL MURAL COMO VEHICULO NARRATIVO E IDENTIDAD CIUDADANA
}

\author{
Otniel López Altamirano \\ Universidad Autónoma "Benito Juárez de Oaxaca", UABJO. México \\ Doutorando, Universidade Estadual Paulista, UNESP. \\ otniel_digital@hotmail.com \\ Mônica Moura \\ Universidade Estadual Paulista, UNESP. \\ monicamoura.design@gmail.com
}

\begin{abstract}
Resumo: El desarrollo del muralismo a lo largo de cada década ha sido objeto de diferentes temáticas y formatos que finalmente constituyen una envolvente sobre muros en espacios interiores y mayormente exteriores, que son la interface comunicativa de gran y rápida difusión. Entre las principales características de este tipo expresión plástica, contiene en ella una especie de relato, es decir, se suceden acciones y situaciones en un mural, lo que vulgarmente también se denomina como película quieta. El documento presenta un levantamiento descriptivo de una serie de murales configurados en diferentes puntos de la ciudad de Philadelphia, en los Estados Unidos, dirigidos por profesionales en las áreas de diseño y artes visuales, como parte del proyecto urbano: El "Programa Mural de la Ciudad de las Artes de Philadelphia", que ha unido a artistas y comunidades a través de un proceso de colaboración, con raíces en las tradiciones de la pintura mural, para crear el arte que transforma los espacios públicos y las vidas individuales de cada uno de que los que participan, interpretando así la identidad asociativa entre los habitantes y el desarrollo del diseño.
\end{abstract}

Palavras-chave: Design, Narrativa, Mural, Identidad, Ciudad.

Abstract: The development of mural painting over each decade has been
the subject of different themes, techniques and formats that ultimately
constitute an envelope or skin on walls mostly indoors and outdoors, which
are highly communicative interface and rapid spread. Among the main
features of such artistic expression, it contains in it a kind of story, that is,
actions and situations occur in a mural, which also commonly referred to as
still film. The paper presents a descriptive survey of a series of murals
configured in different parts of the city of Philadelphia, in the United States,
led by professionals in the areas of design and visual arts, as part of the
urban project: the "Mural Program city of Arts Philadelphia", which has
joined artists and communities through a collaborative process, rooted in
the traditions of mural painting to create art that transforms public spaces 
and individual lives of each of those involved, thus interpreting the associative identity among the inhabitants and design development.

\section{INTRODUCCION}

El mural ha sido uno de los soportes más difundidos de la historia del arte, a pesar de algunas consideraciones más formales, se designa el término de mural a aquella imagen que usa como soporte de sí un muro o pared. La pintura sobre muros predominó principalmente durante la época romana, después decayó durante el tiempo gótico, ya que se suplantaron las paredes por vidrieras, tiempo después volvió con fuerza en el renacimiento. Entre las principales características de este tipo de pintura, contiene en ella una especie de relato, es decir, se suceden acciones y situaciones en un mural, lo que vulgarmente también se denomina como película quieta. ${ }^{1}$ La monumentalidad de la imagen que permite romper el espacio plano del muro es una de las características más sobresalientes. Ver figura 1.

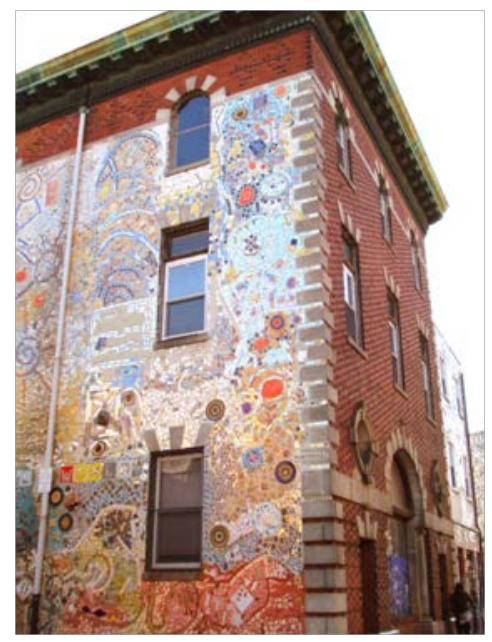

Figura 1- Mural de técnica mixta.

Fuente: Elaborada por el autor, con base a la investigación realizada. Philadelphia, E.U.

Se presenta al mural como el dispositivo que comunica mediante una serie de patrones un cumulo de datos, referencias, diálogos, discusiones, sucesos y entre otros argumentos, el vehículo portavoz de las expresiones del individuo así como su carácter social que lo identifica como un ser pensante, racional, con emociones, errores, que cambia constantemente, que se mezcla entre diferentes estratos y se compromete a preservar la tolerancia en sus variadas aristas, esto es parte de lo que caracteriza al habitante que vive alrededor de un contexto contemporáneo, en una urbe que crece continuamente sin dejar atrás ese hilo histórico que le ha dado origen.

"La identidad no es revelada como algo que necesite ser inventado y no únicamente descubierto" (Bauman, Zygmunt 2005), "No son entidades escondidas en algún lugar

\footnotetext{
${ }^{1}$ Definición de Mural. Concepto definición en $A B C$. Definición ABC. Disponible en internet en http://www.definicionabc.com/general/mural.php Acceso en mayo 2015.
} 
secreto y profundo y sí, algo que necesita ser creado, o en la terminología del Diseño, algo que necesita ser proyectado" (Bonsiepe, Gui 2001).

\section{DESARROLLO}

\section{PHILADELPHIA CON ARTE}

Levantamiento descriptivo de murales "La Milla del Mural".

Situada en el estado de Pensilvania en los Estados Unidos, la ciudad de Philadelphia es conocida como la cuna de la democracia estadounidense, donde se aprobó la Declaración de Independencia el 4 de julio de 1776, desde entonces ha sido escenario de diversos acontecimientos históricos, sociales que giraron hacia los ámbitos culturales y artísticos, este sitio proporciona un modelo viviente de la historia para las generaciones que viven y experimentan dentro de la misma.

"Philadelphia con arte" fue la campaña de marketing de apoyo a la ciudad, que la coloca en la escena de las artes visuales y muchos artistas la convierten constantemente en un lienzo de colores. La campaña altamente integrada, obtuvo un excelente resultado en su primera fase, ya que se contó con la colaboración de entre 10 organizaciones de la ciudad y organismos socios culturales. La abundancia de obras de arte público va más allá de la definición tradicional del arte. ${ }^{2}$

La misión de este proyecto urbano: El Programa Mural de la Ciudad de las Artes de Philadelphia une a artistas y comunidades a través de un proceso de colaboración, con raíces en las tradiciones de la pintura mural, para crear el arte que transforma los espacios públicos y las vidas individuales.

La ciudad de Philadelphia figura como uno de los sitios mas representativos de los Estados Unidos de América, debido a la carga histórica, social, política y emocional de sus habitantes. Ver figura: 2.

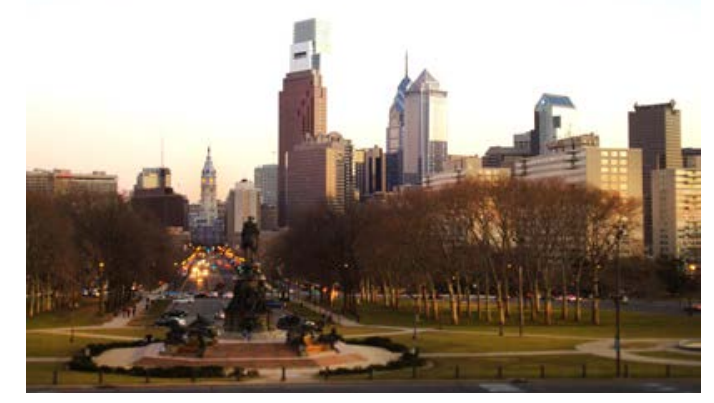

Figura 2. "Ciudad de Philadelphia".

Fuente: Elaborada por el autor, con base a la investigación realizada. Centro de la Ciudad Philadelphia, E.U.

\footnotetext{
${ }^{2}$ Murales de Arte de la Ciudad de Philadelphia. Misión | Programa de Murales de Arte | Disponible en internet en: http://muralarts.org Acceso en mayo 2015.
} 
Paisaje urbano de la ciudad de Philadelphia, que figura como un de los sitios mas representativos de los Estados Unidos de América.

Vio nacer y dio origen al país norteamericano, además es reconocida porque en su ayuntamiento se firmó, en 1786, la primera constitución de Estados Unidos y por ser cuna de la búsqueda del respeto e igualdad de las razas y clases sociales, estableciendo documentos para la equidad de la ciudadanía norteamericana.

\section{"El legado"/ Legacy}

Sarantitis Josh (2005) ha llevado a cabo creaciones monumentales a nivel profesional en los espacios públicos a lo largo de más de 20 años. Sus 40 obras de encargo incluyen las instalaciones de vidrio y mosaicos a nivel regional y en el extranjero, incluyendo San Francisco, Chicago, Philadelphia, Cleveland, Los Ángeles, Minneapolis, Phoenix y Tucson. Ha tenido la capacidad para colaborar con éxito con los profesionales del diseño, administradores de las artes y la comunidad que además tienen sus raíces en el deseo de encontrar un lenguaje común entre la vida cotidiana y las artes. Josh abarca el uso de la tecnología como una herramienta para ayudar a los medios de comunicación combina el deseo de aprender con la ejecución de un técnico calificado.

Las obras incluyen un proyecto de gran envergadura, bajo el titulo de "Legado", de la ciudad de Philadelphia que combina 4.000 metros cuadrados de baldosas de vidrio en un mosaico realista. Escuelas públicas y estudiantes de toda la ciudad, colocaron un millón de piezas de vidrio, utilizando "Tilepile", un programa informático desarrollado por el artista. El programa fue creado específicamente para traducir el diseño de píxel a píxel en el mural con la paleta de vidrio de color de manera eficiente y dentro del presupuesto.

La pregunta que impulsa la práctica de Josh es la forma de crear obras de arte que incluye a la opinión pública. Muchos de sus proyectos incorporarán componentes de la comunidad que permiten el aprendizaje práctico, desmitificando el proceso y el producto para el público. Este artista no tiene miedo a tomar riesgos en la búsqueda de soluciones de diseño, incluyendo la creación de metodologías de carácter técnico, y se siente cómodo trabajando en todos los aspectos de una comisión, incluyendo el sitio de ingeniería, fabricación e instalación. "La experiencia de vida" en combinación con la sensibilidad arquitectónica, el uso de nuevos medios de comunicación y la tecnología, han permitido que las obras públicas de arte hablen hacia un público más amplio. $^{3}$

El diseño de este mural cuenta con una joven afroamericana, una moneda abolicionista y las tablas de un barco de esclavos, que se mezclan con las rayas de la bandera norteamericana, conforme figura abajo.

\footnotetext{
${ }^{3}$ Sarantitis, Josh. Disponible en internet en: http://joshsarantitis.com/ Acceso en mayo 2014.
} 


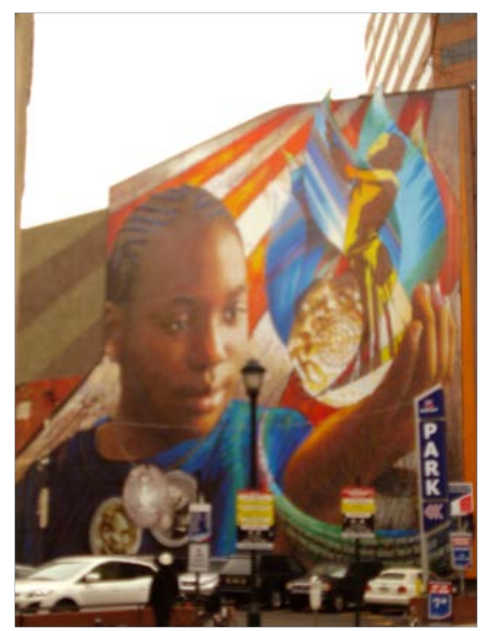

Figura 3- Mural "El legado / Legacy".

Fuente: Elaborada por el autor, con base a la investigación realizada. Philadelphia, E.U.

\section{"La progreso de la gente hacia la igualdad" / A People's Progression Toward Equality}

Nuevamente el artista Sarantitis Josh trabajó en conjunto con el Programa de Murales de Arte de Philadelphia, numerosas organizaciones comunitarias y corporaciones para crear murales públicos en toda la ciudad. Ha establecido una estrecha colaboración con estudiantes y profesores de la Escuela Milton Hershey para crear un gran mural a escala que detalla la historia de 100 años de esta escuela además de crear murales para el público de Philadelfia, creó y supervisó un mural de 9.000 pies cuadrados para el Grupo Lincoln Financial en colaboración con la Corporación Interpark y Weingart Associates Inc. Para producir una pieza de arte público en el estacionamiento Interpark. ${ }^{4}$

Encargado por el Lincoln Financial Group y el Programa de Murales de Arte de Philadelphia, las imágenes plasman a Abraham Lincoln como un mascarón de proa y el catalizador de un movimiento histórico que acabó con la esclavitud y se esforzó por crear una sociedad racialmente con igualdad, ver figura 4.

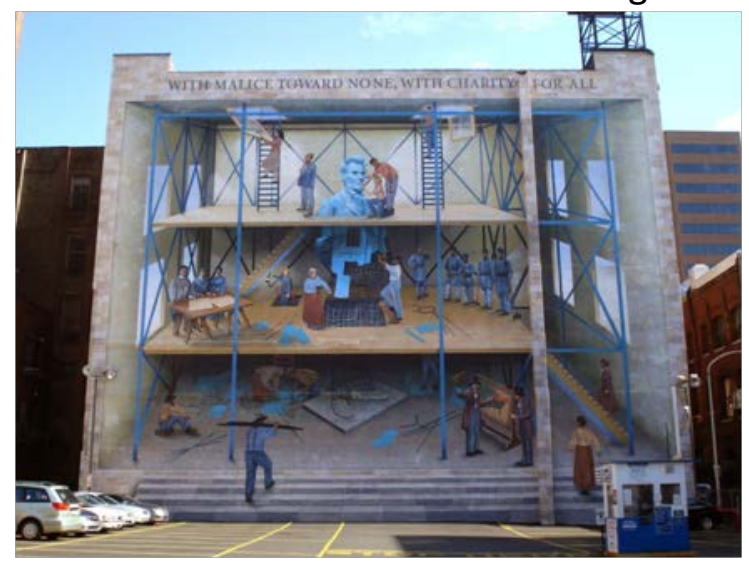

Figura 4- Mural "La progreso de la gente hacia la igualdad”.

Fuente: Elaborada por el autor, con base a la investigación realizada. Philadelphia, E.U.

\footnotetext{
${ }^{4}$ Kim, Senior. Los murales de pintura de arte: muralist.html Acceso en marzo 2014.
} 


\section{"En búsqueda del hogar"/ Finding Home}

En palabras del propio Josh Sarantilis (2008) comenta: "Un amigo me dijo el otro día que si pones un pez bebé en una pecera pequeña, no va a seguir creciendo... ya que debe ser grande el tanque para ofrecerle la posibilidad de seguir creciendo"., esto lo comento a razon de hace unos dos años, fue invitado por el Mural Arts Program para colaborar con el artista, Sarantitis Josh, y los asistentes Shelby Donnelly y Tull Al para crear este gran mural a escala tejido y pintado al aire libre (con otros detalles aplicando una variedad de técnicas que incorporan la impresión, hoja de metal y cable), conforme figura 5 , a seguir.

El objetivo del proyecto fue dar cuenta de las cuestiones relacionadas con la falta de vivienda. A lo largo de muchos meses, Shelby orquestó talleres de tejido en albergues para indigentes, refugios y espacios públicos a lo largo de Philadelphia, donde se tejían las alfombras que después se convertirían en el 'lienzo' para el mural con una sección de 12 pies x 50 pies. El objetivo era reunir a las personas con y sin un hogar, logrando un proceso para crear arte y la conversación en conjunto, teniendo como resultado el reconocer que la hermana, padre, madre, hermano, hija, hijo y otros ayudaron a romper el estigma que rodea la falta de vivienda.

Los antiguos alumnos y profesores del Instituto Kathryn Panenpacker presentaron el mural "Hallazgo del hogar", que fue la génesis del mural "En búsqueda del hogar" realizado con personas sin hogar y con Arts Studio Street Textil. ${ }^{5}$

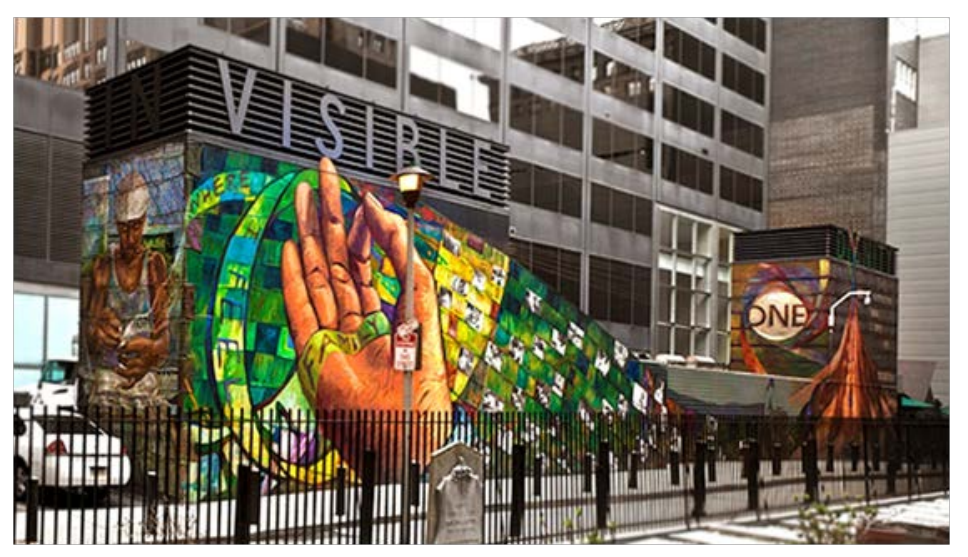

Figura 5- Mural "En búsqueda del hogar"

Fuente: Elaborada por el autor, con base a la investigación realizada, Philadelphia, E.U.

\section{"Las musas de Philadelphia"/ Philadelphia Muses}

Nacida en Olean, Nueva York, Meg Saligman (2000) realiza su primer mural en Philadelphia, Pensilvania. Desde entonces su talento ha florecido en una carrera de reconocimiento internacional. Meg ha creado obras con referentes históricos de Philadelphia "Common Threads" en las calles Broad y Jardín Primavera. Otros trabajos conocidos son "Las Musas Philadelphia", con forme a figura 6, en las calles 13 y Locust,

\footnotetext{
${ }^{5}$ Dedicacion a En busca del hogar. Built a Bridge. Disponible en internet en: http://www.buildabridge.org/home/about-us/ Acceso en marzo 2014.
} 
un reproductor multimedia "Teatro de la Vida" en las calles de Lombard y el nuevo "Passing Through". El trabajo de esta artista se puede ver tanto en el ámbito nacional e internacional del arte público.

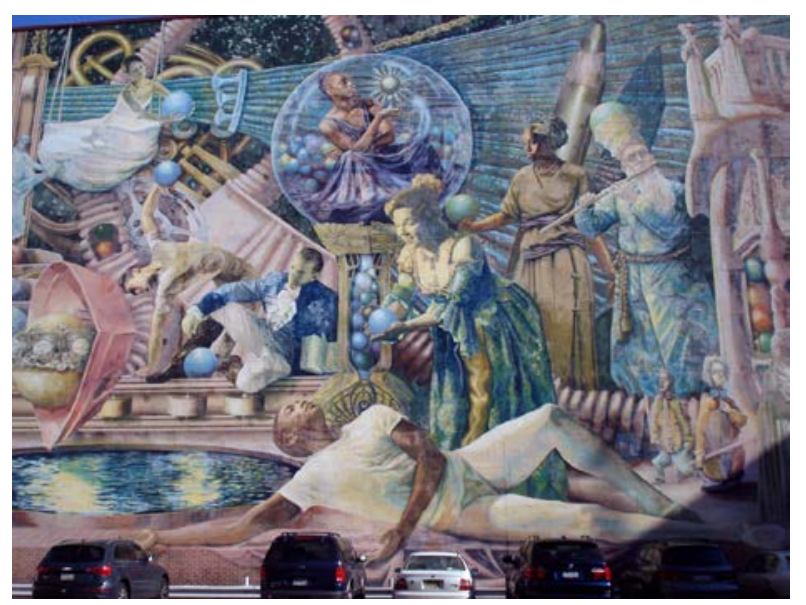

Figura 6- Mural “Las musas de Philadelphia”

Fuente: Elaborada por el autor, con base a la investigación realizada. Philadelphia, E.U.

Desde un principio su trabajo ha sido muy influyente en Philadelphia, ya que ha sido de las primeros artistas que incluyen miembros de la comunidad para la realización del mural en sí. Una de las mayores innovaciones de Saligman fue que ideó un sistema donde la gente de las comunidades fácilmente podían pintar sus propios murales. Esta técnica ha permitido a ciudades enteras e incluso comunidades de prisioneros a participar en el proceso de la realización de arte público. Saligman fue la primer artista en utilizar un ordenador en el diseño de los murales en Philadelphia, su influencia continúa a medida que se han desarrollado muchas técnicas de computación las cuales se utilizan ampliamente en el proceso de diseño. En la actualidad está desarrollando obras digitales que incorporan imágenes de LED y en movimiento. ${ }^{6}$

\title{
"Orgullo y Progreso"/ Pride and Progress
}

Ann Northrup (2002) cree que el simbolismo del arte y tiene una forma única de influir en las personas.

\begin{abstract}
"Siento que el arte tiene un poder especial para llegar al subconsciente y afectan a la gente en un nivel más profundo, a veces sin que ellos lo vieran. Creo que se ven afectados por estas asociaciones, incluso si no se dan cuenta de las técnicas de cómo se hacen las asociaciones visuales." (Ann Northrup, 2002)
\end{abstract}

El mural tiene presente el tema de la diversidad y los derechos de la comunidad gay, muestra una narrativa del trabajo de consignas que se han llevado a cabo en la

\footnotetext{
${ }^{6}$ Saligman, Meg. Murales y Arte de Philadelphia. Disponible en internet en: http://www.megsaligman.com/ Acceso en mayo 2014.
} 
búsqueda de la igualdad y dignidad para la comunidad gay, expresada en el ámbito político y social. Ver figura 7.

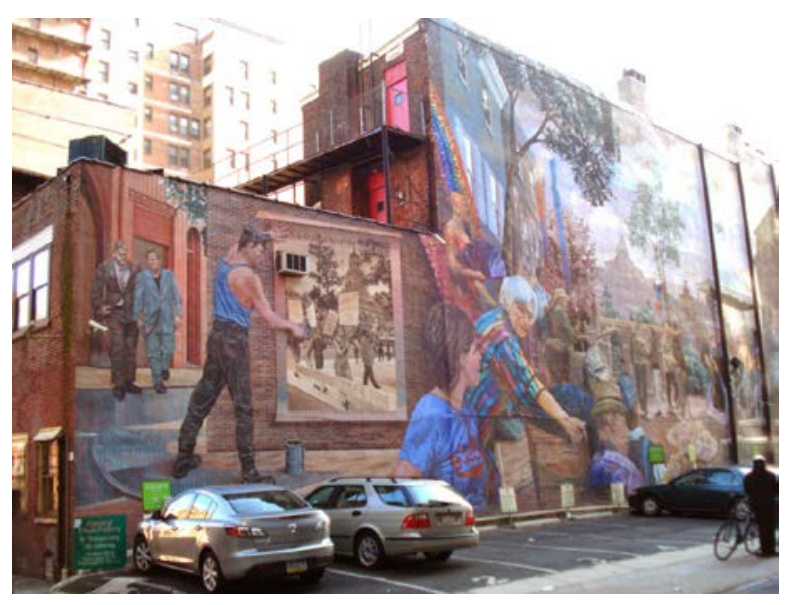

Figura 7- Mural del "Orgullo y progreso".

Fuente: Elaborada por el autor, con base a la investigación realizada. Philadelphia, E.U.

La escena plasma un festival del orgullo gay con referencia a los derechos civiles de la década de 1960, principalmente las marchas en Philadelphia. La figura de la señora mayor usando gafas y mirando fijamente al observador es el punto central del mural. Esta ha sido la ciudad de primicias para muchas cosas en toda su historia. El mural se convirtió en el primer proyecto de lesbianas, arte público gay, bisexuales y transexuales en el país, después de sus inicios con carga social y política.

\section{“Otoño"/ Autumn}

Propuesta de mural con un tema sobre vegetación, nuevamente por el artista David Guinn (2012) plasma a una temporada del año, donde los pigmentos aplicados han sido rojisos, sepias, naranjas y ocres. El uso de la cuadricula del pixel es mayormente visible en el follaje de los arboles, que dejan entrever la proyección del suelo en bloques de colores a mayor escala, además el artista incluyo elementos naturales, como el cause de un rio y demás animales representados en un contexto campestre. Este mural se extiende libremente a lo largo de la fachada del inmueble y parte de un estacionamiento publico. Ver figura 8.

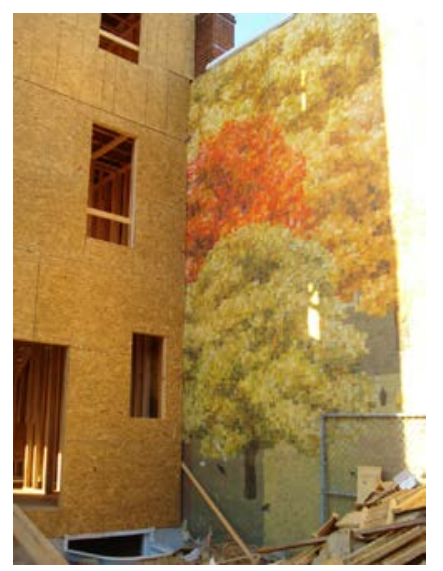

Figura 8- Ultima fracción del mural de "Otoño" que aun queda visible al público y vecinos.

Fuente: Elaborada por el autor, con base a la investigación realizada. Philadelphia, E.U. 
Exsitió una gran polémica entorno al mural de Otoño, de este artista ya que ahora narra una historia diferente: La obstrucción inminente, por una vivienda de reciente construcción, que afectó totalmente la visibilidad de esta pieza tan apreciada por los vecinos y visitantes.

En palabras del propio Guinn, que da su opinión para el sitio web de Noticias Reseña del sur de Philadelphia: "Estoy muy triste por el mural que ha quedado cubierto", "Es mi mural favorito que he pintado, era una parte de mí." "La lucha de los vecinos que se levantaron en contra de la construcción de una vivienda que cubre ahora al mural fue impresionante". Residentes de Bella Vista recaudaron miles de dólares para tratar de comprar el lote de los desarrolladores de la construcción.

"Parte del mural seguirá siendo visible desde detrás de la casa", dice, "para refrescar la memoria de lo que originalmente fue." 7

\section{CONCLUCION}

Cada una de las expresiones plásticas arriba presentadas, son parte de toda una serie de 17 murales, en un cuadrante central de la ciudad, extendiéndose en un recorrido que invita a hacer una lectura del contexto inmediato de cada uno de los predios donde fueron plasmadas las escenas que son parte de un discurso proyectual. El entusiasmo de realizar el registro de cada pieza, ha sido en la búsqueda de proyectos ejecutados con construcciones narrativas que proponen diálogos apoyados en elementos visuales de gran escala, de apreciación, uso y participación pública, en sincronía con la intervención en la ciudad y de órganos gubernamentales.

Esta ciudad ha apostado hacia una línea multicultular, de diversidad de genero y raza, como resultado de todas esas vertientes que se han extendido desde décadas anteriores, quizás en una sincera manifestación de disculpa a todo aquel ciudadano que ha sido lastimado o que sigue siendo susceptible ante todas aquellas expresiones de intolerancia, de ahí que el gobierno actúa del modo que sería "políticamente correcto".

Resulta ser reconocida la trascendencia del proyecto "La Milla del Mural", ya que es palpable el trabajo multidisciplinario, entre profesionales de las artes y de diseño, benefactores que aportaron los recursos para llevar a cabo y todos aquellos que contribuyeron en la intervención en mayor o igual medida en cada una de las piezas. Por último y aun más importante, el interés de los grupos ciudadanos, es en este punto donde habrá que recalcar con mayor énfasis, que gracias a esta estrategia se obtuvo como resultado un compromiso simbólico entre individuo- proyecto- ciudad. Bajo este conjunto de engranes se entiende que fue minuciosamente la selección aleatoria de las calles, edificios que cumplieran con características muy especificas con base al uso

\footnotetext{
${ }^{7}$ Myers, Joseph. Programa de Murales de Arte, "Otoño" bajo ataque | Noticias | Reseña del sur de Philadelphia. Revisión del sur de Philadelphia | Las últimas noticias locales y opiniones. Disponible en internet en http://www.southphillyreview.com/news/Mural-Art-Programs-Autumn-under-attack132660533.html Acceso en marzo 2014.
} 
de suelo, las dimensiones del mismo, las fachadas visibles o colindantes, la accesibilidad visual para los observadores, el numero de elementos ornamentales con los que contaban, entre quizás muchas otras consideraciones. Los vecinos que formaron parte de estos proyectos contribuyeron algunos de manera directa y otros posterior a la ejecución, siendo hoy en día los vigilantes y protectores de los murales que se encuentran en su calle, de modo que cualquier acto de tipo vandálico o agresión a una de estas piezas, actúan de manera protectora y se manifiestan por su conservación. Es de este modo que los murales narrativos, se han tornado parte de si mismos, cada uno los representa y les atribuyen una distinción, son los punto de referencia en el plano de la ciudad.

Las posibilidades de extender este tipo de ejercicio consolidado pueden potencializar el desarrollo cultural y de identidad en la construcción de imagen urbana y distintivos populares, un ejemplo más de la labor del diseño llevando de la mano un discurso social.

\section{REFERENCIAS}

Definición de Mural. Concepto definición en ABC. Definición ABC. Disponible en internet en http://www.definicionabc.com/general/mural.php Acceso en mayo 2015.

Murales de Arte de la Ciudad de Philadelphia. Misión | Programa de Murales de Arte | Disponible en internet en: http://muralarts.org Acceso en mayo 2015.

Sarantitis, Josh. Disponible en internet en: http://joshsarantitis.com/ Acceso en mayo 2014.

Kim, Senior. Los murales de pintura de arte: Michael Webb, muralista arquitectónico. Murales de Arte Pintura. Disponible en internet en:

http://kimsenior.blogspot.mx/2008/01/michael-webb-architectural-muralist.html Acceso en marzo 2014.

Dedicacion a En busca del hogar. Built a Bridge. Disponible en internet en: http://www.buildabridge.org/home/about-us/alliances/alliance-postings/item/154finding-home-mural-dedication Acceso en marzo 2014.

Saligman, Meg. Murales y Arte de Philadelphia. Disponible en internet en: http://www.megsaligman.com/ Acceso en mayo 2014.

Muller, A. I. Conozca al muralista: Un perfil de Ann Northrup. Comité de servicio de los amigos americanos | Comité de valores en accion. Disponible en internet en: http://afsc.org/story/meet-muralist-profile-ann-northrup Acceso en mayo 2014.

Myers, Joseph. Programa de Murales de Arte, "Otoño" bajo ataque | Noticias | Reseña del sur de Philadelphia. Revisión del sur de Philadelphia | Las últimas noticias locales y opiniones. Disponible en internet en http://www.southphillyreview.com/news/MuralArt-Programs-Autumn-under-attack-132660533.html Acceso en marzo 2014. 